\title{
Silicon Thermal Flow-Sensor Semi-ideal Model
}

\author{
U. Hammerschmidt ${ }^{1}$ (D) C. Sosna ${ }^{2} \cdot$ A. Benkert ${ }^{3}$
}

Received: 10 December 2021 / Accepted: 17 January 2022 / Published online: 5 February 2022

(c) The Author(s) 2022

\begin{abstract}
A so-called semi-ideal model has been derived for a pulse-operated MEMS thermal flow sensor by reciprocal numerical mapping of the ideal model of an instantaneous line heat source to the experimental data of the sensor. The novel model not only applies as a precise potential working equation for the sensor but also provides insight into the complete thermal signal transmission chain from the heater to the thermometers of the sensor. It gives answers to prominent peculiarities of the actual heat path of the sensor, prepares ways to improve its design, and offers guidance for a better control of the measuring process. The semi-ideal model was derived in order to replace the so far utilized empirical relations that require multiple calibration measurements. It was found out that the transient temperature profile of the sensor in response to an electrical pulse to the heater is substantially shaped by series thermal conduction of heat. Moreover, it is demonstrated that the impact of the thermal mass of a (practical) line heat source on its transient temperature and released enthalpy can be modeled by considering a virtual cylinder surrounding the source. The same analytical technique also applies for (practical) temperature stations. The findings of the study primarily concern the MEMS sensor. Nevertheless, the presented simple method of numerical mapping can be an effective analytical tool for mathematical modeling by means of numerical data analysis and function evaluation. The concept of a virtual cylinder can help in modeling the dynamics of practical pulsed heating.
\end{abstract}

Keywords Instantaneous line heat source $\cdot$ MEMS $\cdot$ Numerical mapping $\cdot$ Practical pulsed heat source $\cdot$ Semi-ideal model $\cdot$ Thermal flow sensor $\cdot$ Transient temperature response $\cdot$ Working equation

U. Hammerschmidt

ulf.hammerschmidt@outlook.com

1 Physikalisch-Technische Bundesanstalt, Bundesallee 100, 38116 Braunschweig, Germany

2 Diehl Metering GmbH, Donaustrasse 120, 90451 Nürnberg, Germany

3 Diehl Metering GmbH, Industriestrasse 13, 91522 Ansbach, Germany 


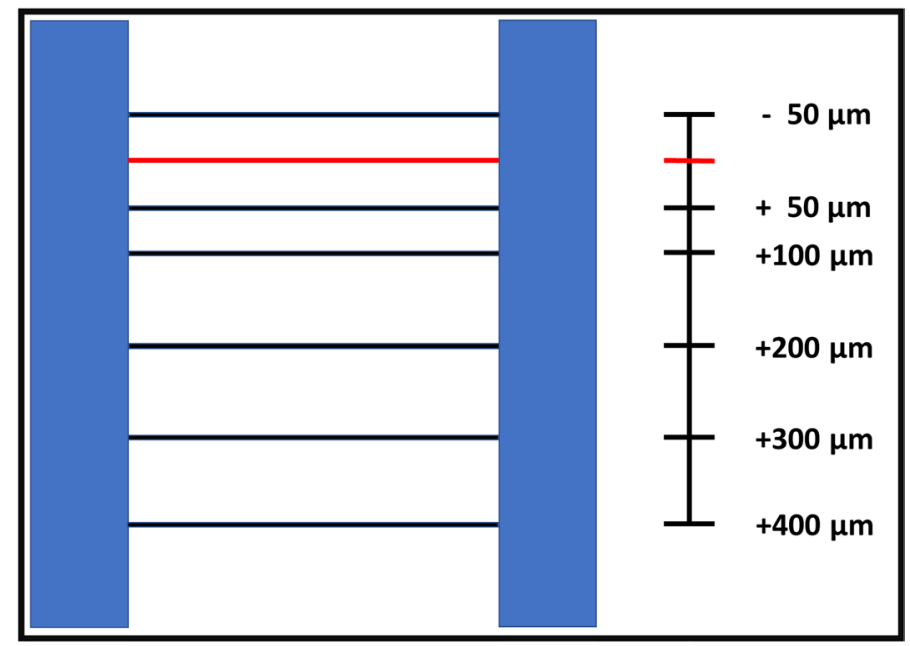

Fig. 1 Schematic top view of the MEMS PTF sensor. Seven microbeams, each of $2000 \times 6 \times 0.3 \mu \mathrm{m}^{3}$, traverse the etched trench $\left(2000 \times 2500 \times 300 \mu \mathrm{m}^{3}\right)$. (from top: thermometer TL050 $(-50 \mu \mathrm{m})$, heater $\mathrm{H}$ $(0 \mu \mathrm{m})$, thermometers T050 $(+50 \mu \mathrm{m}), \mathrm{T} 100(+100 \mu \mathrm{m}), \mathrm{T} 200(+200 \mu \mathrm{m}), \mathrm{T} 300(+300 \mu \mathrm{m})$, and T400 $(+400 \mu \mathrm{m}))$

\section{Introduction}

In two previous reports by the same authors, a single-short-pulse thermal flow sensor on a chip has been introduced [1,2]. This so-called pulsed thermal flow (PTF-) sensor is able to precisely determine the flow velocity, $v$, of gases at a standard uncertainty of $1.5 \%$. The sensor can be operated in two distinct modes of operation: "conventional downstream" [1] and "advanced upstream" [2]. In the latter mode, the amplitude of the peak of the temperature response, $\Delta T_{\max }(v)$, is determined as the measurand. In the downstream method, it is the instant in time of a peak, $t_{\max }(v)$. The objective of the present experimental, numerical, and theoretical study is to create an analytical model of the transient temperature behavior of the sensor to replace the existing two mode-specific empirical data reduction equations.

The PTF sensor is realized in MEMS-technology ${ }^{1}$ and only consists of seven similar electrical resistors. These are arranged on separate parallel microbridges traversing a $2 \mathrm{~mm}$ wide trench etched into the silicon substrate (Fig. 1). One of the resistors is operated as a Joule heater, the others act as temperature stations. To measure flow velocities, only one upstream or one downstream station is required. So far, the remaining four thermometers are of study purposes only. They were used to examine local transient temperature responses of a quiescent gas to an electrical pulse fed to the heater.

Despite its plain design and its two fairly simple modes, until now, it was not possible to model the PTF sensor completely analytically and at sufficient precision and

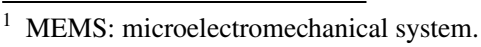


Table 1 Thermal conductivity, thermal diffusivity, and volumetric-specific heat capacity for silicon, the silicon-nitride films, and nickel at $23{ }^{\circ} \mathrm{C}[4,5]$

\begin{tabular}{llll}
\hline Material & $\begin{array}{l}\text { Thermal conductivity } \\
{\left[\mathrm{W} \cdot \mathrm{m}^{-1} \cdot \mathrm{K}^{-1}\right]}\end{array}$ & $\begin{array}{l}\text { Thermal diffusivity } \\
{\left[\mathrm{mm}^{2} \cdot \mathrm{s}^{-1}\right]}\end{array}$ & $\begin{array}{l}\text { Vol. spec. heat } \\
{\left[\mathrm{MJ} \cdot \mathrm{m}^{-3} \cdot \mathrm{K}^{-1}\right]}\end{array}$ \\
\hline Silicon & 168 & 87 & 1.93 \\
Silicon-nitride film & $\approx 2.1$ & $\approx 1.5$ & 1.40 \\
Nickel (98.7) & 72 & 19.5 & 3.69 \\
\hline
\end{tabular}

rigor. In addition to the existing mode-specific empirical working equations, a fit function for the transient output signal of the device is available. It reliably predicts both measurands, $\Delta T_{\max }$ and $t_{\max }$. This regression model is formally based on the best suited closed-form solution at hand of Fourier's second law [3]. The respective pulse function works with a remarkably high goodness of fit $\left(R^{2} \geq 0.98\right)$ but only consists of parameters that do not directly represent the crucial physical attributes of the gas under test. Early attempts to generate relationships between the purely numerical fit-parameter set and the actual measurands revealed that the transient temperature response of the PTF sensor is too complex to be modeled within the idealized scenario of the selected heat equation solution.

Here, a new experimental, numerical, and theoretical attempt is made to implement, as far as possible, the evident non-ideal physical characteristics of the sensor into the basic ideal heat conduction model. The objective is a semi-ideal model to predict the transient output signals of the sensor precisely. For this purpose, the imperfections of the sensor first had to be explicitly disclosed and then translated into tractable mathematical expressions. The required further background information on the transient thermal behavior of the sensor could be obtained from specific experiments and 3-dim. finite element method (FEM) simulations, all are described and discussed in this report.

First, the ideal model solution of interest here is briefly reconsidered followed up by a concise introduction to the closely related regression model. The ideal model solution on one hand and typical experimental and numerical (FEM) temperature responses on the other hand are carefully compared to locate the major mutual discrepancies. Hereby, all relevant quantities and their relationships have to be identified. For each quantity, it has to be decided whether the restriction(s) that has/have to be obeyed are soft or hard with respect to the above-mentioned basic discrepancies.

\section{Sensor Design and Experimental Setup}

The design of the PTF sensor has already been described in detail in [1, 2]. Figure 1 shows a schematic top view of a silicon chip of the current third generation. Seven microbeams, each of $2000 \times 6 \times(0.3+0.2) \mu \mathrm{m}^{3}$, traverse an etched trench of size $2000 \times 2500 \times 300 \mu \mathrm{m}$. Each "bridge" is composed of a $300 \mathrm{~nm}$ thick layer of silicon nitride and a nickel coating $(200 \mathrm{~nm})$ to let this "microwire" operate as a Joule heater ("transient hot-wire") and/or as a resistance temperature detector (RTD). 
The electrical resistance of a nickel film at room temperature is $170 \Omega \pm 15 \%$. The related temperature coefficient of the resistance has been determined experimentally to be $\alpha_{\mathrm{Ni}}=0.005 \mathrm{~K}^{-1}$ (Table 1$)$.

In the following, a rectangular coordinate system is superimposed on the active surface area of the chip (cf. Fig. 1) in such a way that the longitudinal axis of the heater is on the $y$-axis. All centers of the seven closely spaced beams of a chip are on the $x$-axis. The center of the heater is at the origin. Therefore, in the following, the above cylindrical model with $r=\sqrt{x^{2}+y^{2}+z^{2}}$ is slightly reduced to a plane (sub-) model with the preferential direction $\pm x$.

In Fig. 1, the second microbeam from above normally acts as the heater $(x=0 \mu \mathrm{m})$. Right next to either side of this beam are the upstream (uppermost) thermometer TL050 $(x=-50 \mu \mathrm{m})$ and the first downstream thermometer T050 $(x=50 \mu \mathrm{m})$. In the figure below the T050 station, subsequently there are four additional downstream thermometers: T100 $(x=100 \mu \mathrm{m})$, T200 $(x=200 \mu \mathrm{m})$, T300 $(x=300 \mu \mathrm{m})$, and T400 $(x=400 \mu \mathrm{m})$.

For experimental purposes, the PTF sensor is mounted on a printed circuit board and covered by a U-shaped mini-channel made of plastic. The inner cross-section area of the channel is $1.2 \times 0.7 \mathrm{~mm}^{2}[1,2]$. The heater is supplied by a programmable constant voltage source. For a run, one single constant voltage square pulse of $\max .45 \mathrm{~mW}$ is generated and fed to the heater for a period of $30 \mu \mathrm{s}$.

Each RTD is part of a separate Wheatstone-bridge circuit. The corresponding difference voltage of a bridge is amplified by a factor of 25 and fed to a programmable I/O Card consisting of four input channels. Therefore, a maximum of four thermometers can simultaneously be read out. For a run on a gas, the on-board timer is started by the rising edge of the heater pulse. Each run was performed for $10 \mathrm{~ms}$ or $40 \mathrm{~ms}$. Within this period, per channel, $\mathrm{n}=501$ uniformly spaced observations were carried out.

All experiments described here were performed at room temperature $\left(23{ }^{\circ} \mathrm{C}\right)$ and atmospheric pressure (1013 mbar) in quiescent gas.

Free convective and radiative heat transfers during a run can be largely excluded: due to the very short period in measurement time $\left(\Delta t<10 \times 10^{-3} \cdot \mathrm{s}\right)$ and the very small gas volume $\left(V_{G}<2 \times 1.2 \times 0.7 \mathrm{~mm}^{3}\right)$ there is no onset of natural convection. Radiation may be treated as negligible because of the small temperature rise of the heater $\left(\Delta T_{H}<5 \mathrm{~K}\right)[1,2]$.

\section{Theory}

As mentioned above, the PTF sensor can either be operated in "downstream" or in "upstream" mode to determine the flow velocity. In both modes, a very short pulse of a known amount of enthalpy is liberated into the moving fluid where the energy is predominantly transferred by forced convection and, naturally, also by conduction. When operated in "downstream" mode, the time-of-flight, $t_{\max }$, of the pulse from the heater to the next downstream thermometer is measured whereas in "upstream" mode, the maximum amplitude, $\Delta T_{\max }$, of the pulse is the 
measurand by the adjacent downstream station. Therefore, the two key characteristics of a sensor output signal are the function values, $\left(\Delta T_{\max }, t_{\max }\right)$, of the maximum of a pulse.

An analytical model to predict the above two quantities of interest here, first and foremost, has to deal with conduction of heat in fluids. Once the basic transport equation is set up, the effect of forced convection can be incorporated. Here, only the first step of this procedure, finding an adequate solution to Fourier's heat conduction equation, will be presented and discussed.

\subsection{Ideal Model}

The location-, $r$, and time-, $t$, dependent temperature field, $\Delta T(r, t)$, generated by an instantaneous point, line, or plane heat source within in an unbounded homogeneous medium of constant thermal properties is given by [3]:

$$
\Delta T(r, t)=\frac{H_{0}}{(4 \pi a t)^{\mathrm{p}}\left(\rho c_{p}\right)} \exp \left(\frac{-r^{2}}{4 a t}\right) \text { with } \mathrm{p}=\left\{\begin{array}{l}
3 / 2 \text { point source: } H \\
2 / 2 \text { line source: } H / L \\
1 / 2 \text { plane source: } H / S
\end{array}\right\}=H_{0} .
$$

In case of a line source of length, $L$, the liberated specific enthalpy is $H_{0}=H / L$. whereas for a plane source of cross-section area, $S, H_{0}=H / S$ is valid. The fluid is of thermal conductivity, $\lambda$, thermal diffusivity, $a$, and volumetric-specific heat, $\left(\rho c_{p}\right)=\lambda / a$. For reasons of clarity and comprehensibility, the above relation is rewritten to:

$$
\Delta T(r, t)=\frac{A}{t^{\mathrm{p}}} \exp \left(\frac{-B}{t}\right) \text { with } A=\frac{H_{0}}{(4 \pi a)^{\mathrm{p}}\left(\rho c_{p}\right)} \text { and } B=\frac{r^{2}}{4 a} .
$$

As will be justified below, here, a line source is of interest, i.e., $p \equiv 1$ and $H_{0}=H / L$. Consequently, the isotherms are cylindrical (cf. Fig. 3). The respective temperature maximum, the peak

$$
\Delta T_{\max }=\frac{A}{B \mathrm{e}}=\frac{H}{4 \pi L r^{2}\left(\rho c_{p}\right) \mathrm{e}}
$$

occurs at time

$$
t_{\max }=\frac{r^{2}}{4 a}=B
$$

Here, the Euler number is denoted by e $(=2.71828 \ldots)$. In cases of possible confusion, the respective ideal model quantities, e.g., the time of a peak, $t_{\max }^{+}$, are denoted by a superscript "plus" (cf. Table 3).

Due to the conservation of energy, there is a (natural behavior) propagation loss of temperature. From Eqs. 1 and 3, one gets, 


$$
\frac{\Delta T_{\max }\left(r_{2}\right)}{\Delta T_{\max }\left(r_{1}\right)}=\left(\frac{r_{1}}{r_{2}}\right)^{2} .
$$

\subsection{Regression Model}

After the following parametrization,

$$
A^{*}=\frac{H_{0}}{(4 \pi a)^{\mathrm{p}^{*}}\left(\rho c_{p}\right)} \text { and } B^{*}=\frac{r^{2}}{4 a},
$$

the ideal model relation, Eq. 1, can readily be used as a regression function,

$$
\Delta T^{*}=\frac{A^{*}}{t^{\mathrm{p}^{*}}} \exp \left(-\frac{B^{*}}{t}\right) \text { with } \mathrm{p}^{*}=\mathrm{p}
$$

to validate the above ideal model solution for its potential application on the PTF sensor output signal.

For a better formal distinction from the ideal model quantities, the respective regressive model quantities are indicated by a superscript "asterisk."

The peak,

$$
\Delta T_{\max }^{*}=\frac{A^{*}}{\left(B^{*} / \mathrm{p}^{*}\right)^{*} \cdot \mathrm{e}^{\mathrm{p}^{*}}},
$$

occurs at time

$$
t_{\max }^{*}=\frac{B^{*}}{\mathrm{p}^{*}} .
$$

For this study, all non-linear fits were carried out using the Levenberg-Marquard algorithm with the free parameters $A^{*}, B^{*}$, and $\mathrm{p}^{*}$. In principle, if assigned as a free parameter, the fitted numerical value of $\mathrm{p}^{*}$ can help to identify the most probable idealized shape of the heater (cf. Eq. 1).

\subsection{Model Assessment}

In the course of the functionality tests of the thermal flow sensor [1, 2], the following key findings on the ideal and the regression models soon have been made.

\subsubsection{Failure of the Ideal Model}

The simple ideal model of an instantaneous line heat source fails to correctly predict both relevant measurands of the sensor, the amplitude, $\Delta U_{\max }\left(t_{\max }\right) \propto \Delta T_{\max }\left(t_{\max }\right)$, as well as the time, $t_{\max }$ of the maximum. Yet more, as Fig. 2 exemplifies, the experimental T050 output voltage signal for surrounding quiescent $\mathrm{N}_{2}$ clearly differs from the corresponding transient of the ideal 


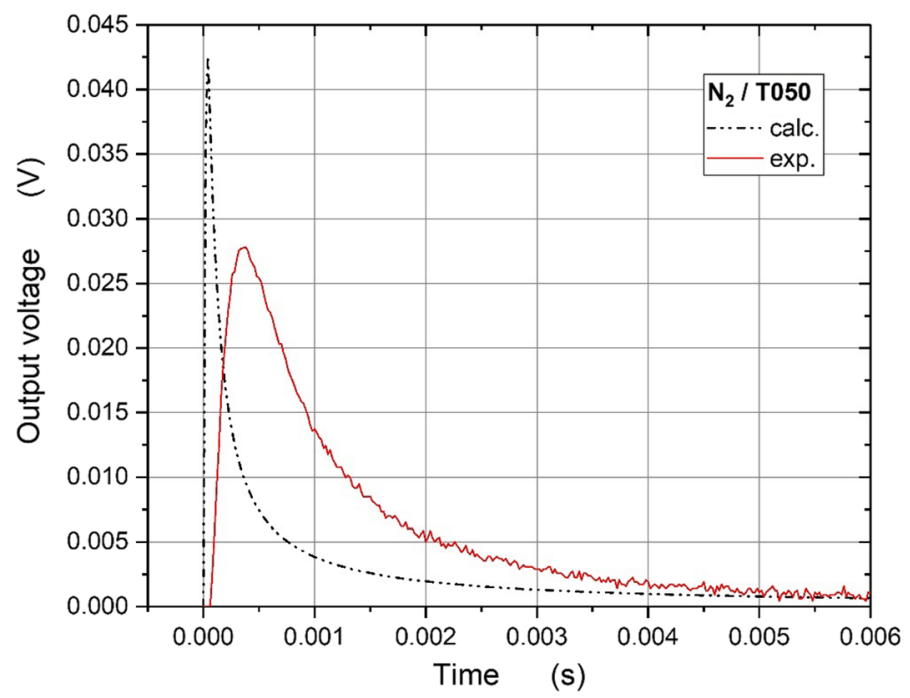

Fig. 2 Experimental and theoretical transient temperature responses to a $30-\mu$ s electrical heater pulse at $r=50 \mu \mathrm{m}(\mathrm{T} 050)$ in quiescent $\mathrm{N}_{2}$. The theoretical curve is calculated according to the ideal model based on the experimental input data (see text)

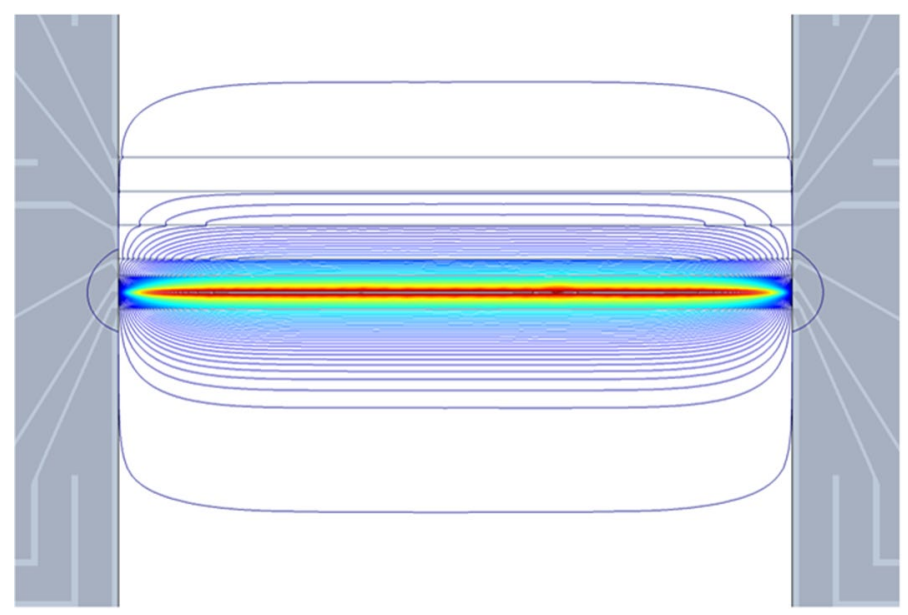

Fig. 3 FEM topview of the sensor (top microbeam: T400) showing the isotherms of the transient temperature response to a heater step pulse of $2 \mathrm{~V}$ within nitrogen after $1 \mathrm{~ms}$

model. This is all the more remarkable since the associated finite element (FEM) simulation of the topview of the sensor furnishes a family of near-cylindrical ${ }^{2}$

\footnotetext{
${ }^{2}$ Characteristic departures from the cylindrical shape are due to the boundary conditions at either edge of the trench.
} 


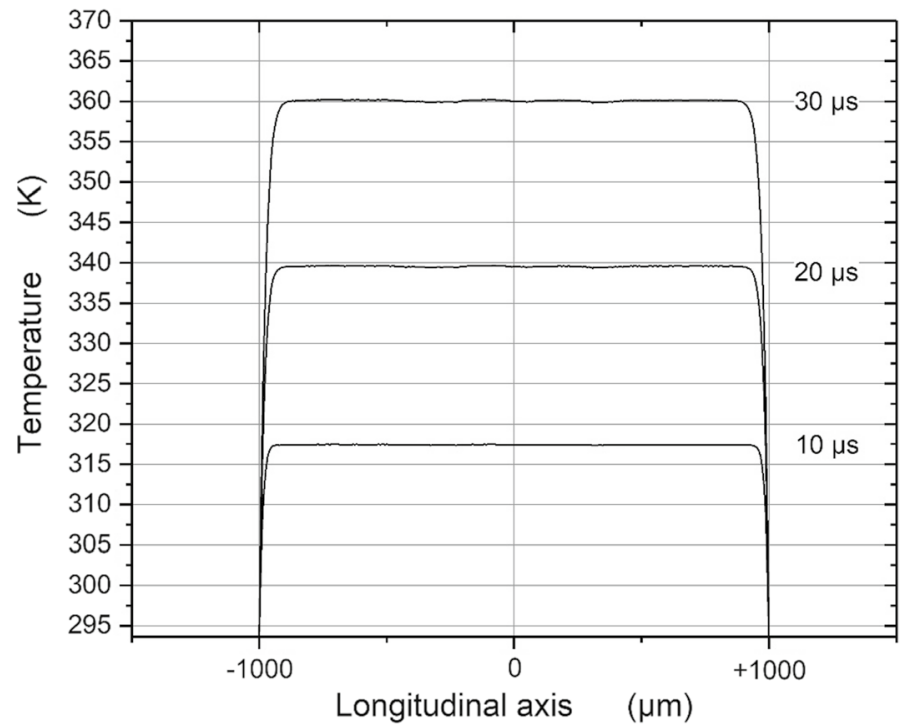

Fig. 4 FEM simulation of the temperature distribution of the heater along its longitudinal axis after $10 \mu \mathrm{s}, 20 \mu \mathrm{s}$, and $30 \mu \mathrm{s}$ in $\mathrm{N}_{2}$. The electrical square pulse to the heater lasted for $30 \mu \mathrm{s}$

isotherms (Fig. 3). The same simulation yielded the temperature profile along the (longitudinal) $y$-axis of the heating resistor of the PTF sensor at three consecutive points in time: $10 \mu \mathrm{s}, 20 \mu \mathrm{s}$, and $30 \mu \mathrm{s}$ after the release of an electrical square pulse of $30 \mu$ s to the heater (Fig. 4). Even at $30 \mu$ s, some $90 \%$ of the central length of the heater is at almost uniform temperature. The input enthalpy to the heater has deliberately been chosen significantly larger than for a standard run of the sensor in order to test a stress scenario under increased workload.

\subsubsection{Malfunction of the Regression Model}

Despite the fact that the ideal model itself falls short, its parameterized version, Eq. 5, the regression model, impresses through a high numerical goodness of fit $[1,2]$ (e.g., Figs. 5 and 6).

However, all three fitting parameters constantly numerically differ from their related ideal model complement, $A^{*} \neq A=H /(4 \pi L \lambda), B^{*} \neq B=r^{2} /(4 a)$ and $\mathrm{p}^{*} \neq \mathrm{p}=1$ (cf. Table 3 ). In both cases of the composite variables, the sought thermal parameters, $H, \lambda$, and $a$, could not be related to the fitting parameters.

A serious consequence of the above inequality of $\mathrm{p}$ and $\mathrm{p}^{*}$ is that Eq. 6 appears dimensionally inhomogeneous and inconsistent in units. A finding that is contradictory to the symmetry conditions of the ideal model. Nevertheless, Eq. 9, derived from Eq. 6, is dimensionally homogeneous because the unit of $\left[B^{*}\right]=\mathrm{s}$ and $\mathrm{p}^{*}$ is of non-dimensional character. 


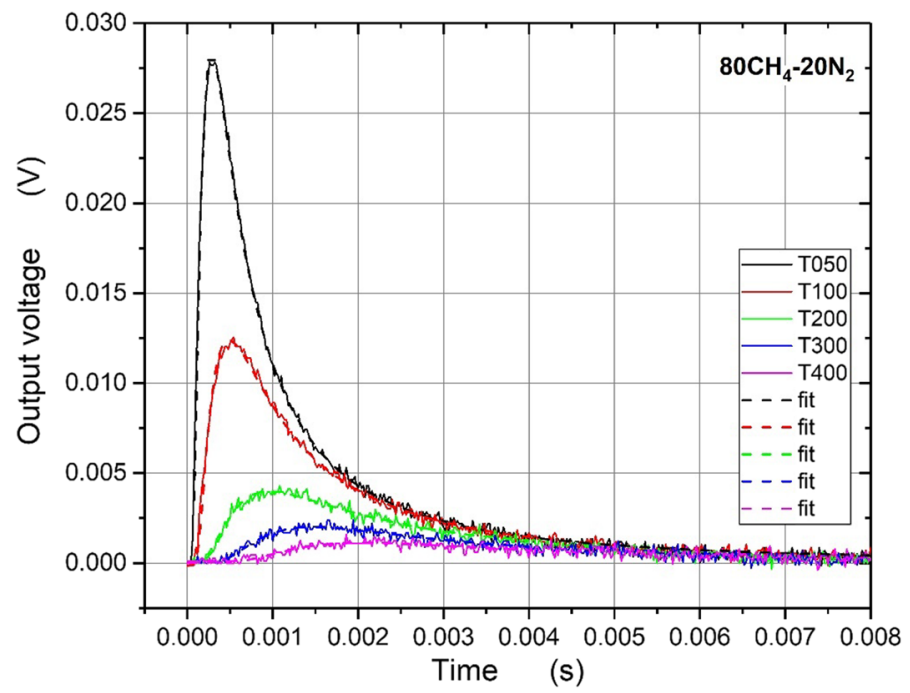

Fig. 5 Experimental transient temperature responses to a $30-\mu$ s electrical heater pulse, observed at distances of $r=50 \mu \mathrm{m}, 100 \mu \mathrm{m}, 200 \mu \mathrm{m}, 300 \mu \mathrm{m}$, and $400 \mu \mathrm{m}$ from the heater in quiescent $80 \mathrm{CH}_{4}-20 \mathrm{~N}_{2}$

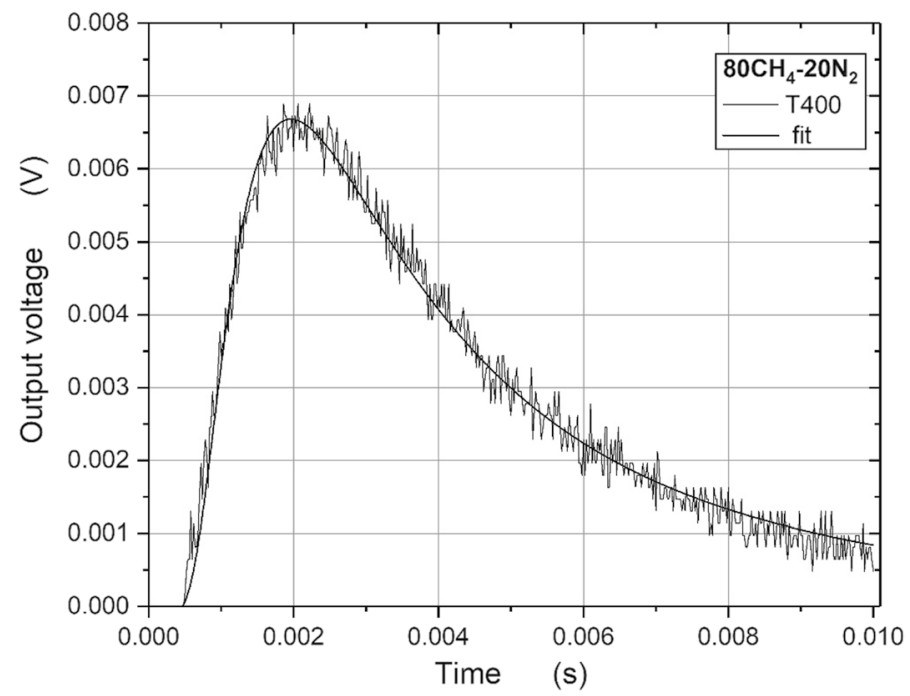

Fig. 6 Output voltage of the T400 thermometer vs. time, shown are the experimental and the regression curves (see Fig. 5)

It is worth noting here that a two-parameter set of $A^{*}$ and $B^{*}$ (for $\mathrm{p}^{*}=\mathrm{p}$ fixed), consistently leaves the above regression function under-parameterized, i.e., it does not converge. 


\subsubsection{Failure Analysis}

The above mismatches were supposed to chiefly result from the following three basic simplifying assumptions to the ideal model:

(1) In the ideal model, an instantaneous line heat source (of infinite length and vanishing radius) liberates a Dirac pulse of a certain amount of enthalpy. In contrast, the resistive heater $(R=170 \Omega)$ of the sensor is cuboid of size $2000 \times 6 \times 0.5 \mu \mathrm{m}^{3}$. It is fed with a $\tau=30 \mu \mathrm{s}$ long electrical square pulse of predefined voltage, $U$, that is converted into a heat pulse of enthalpy, $H=U^{2} / R \cdot \tau$, but significantly different in shape and longer in duration. Due to the non-vanishing thermal mass of the heater, its temperature initially continuously rises to a maximum and then only gradually decays to the starting value.

(2) The ideal-case gas domain is unbounded. In contrast, the gas surrounding the active components of the sensor is contained inside the walls of the tiny test volume between the (micro) trench inside the chip and the mini-channel covering the whole sensor. As soon as an imposed rate of heat flow reaches one of the outer walls of the test volume, heat can easily be conducted to the ambient resulting in a certain loss in the temperature signal of each resistance thermometer of the sensor.

(3) An ideal-case temperature station is of point shape and, thus, massless and of an instantaneous measurement indication. In contrast, the RTDs of the sensor are of non-vanishing volume and heat capacity. Hence, these components will suffer from temperature-dependent individual thermal delays.

\subsection{Methodology}

The suggested solution to the above paradox that the ideal model fails to predict the temperature transients of a PTF sensor whereas the regression model, derived from the ideal model, numerically accurate describes the transient was that the ideal model might be incomplete. This assumption had first to be tested true or false. In principle, there are two different approaches to follow: the analytical and the numerical one.

The first method starts with the appropriate Fourier equation. This partial differential equation has to be solved for a transient instead of an instantaneous and constant enthalpy input, $H(t)$, into a linear heat source with thermal mass in place of a massless source. The latter task involves a convolution of the enthalpy pulse with the Dirac pulse. So far, for such a scenario, there is no closed-form solution at hand. A Laplace transform, here, furnishes a sum of impulse responses that may be truncated to three or four terms. Nevertheless, it turned out that this relation does not accurately predict the temperature transient of the sensor. After having changed the basic approach, it finally was found out that the impact of the thermometers on the propagation of enthalpy might be crucial. This latter effect was not incorporated into the analytical approach. 
The second method, the numerical one, deals with five different (time-)ordered datasets, $n$-tuples, each of $n=501$ members, comprising the experimental and the related theoretical data. These are (1) the sequence of the observation time points themselves, $\langle t\rangle$; (2) the sequence of experimental voltage points, $\langle U\rangle$; (3) the sequence of fitted voltage data according to the regression model, Eq. $6,\langle R\rangle$; (4) the sequence of calculated voltage data according to the ideal model, Eq. $1 ;\langle I\rangle$; and the fifth set presents the solution data, $\langle S I\rangle$, the sequence of voltages calculated from the novel semi-ideal model of the PTF sensor.

The specific procedure used to initially test the above hypothesis true and then to identify, analyze, and compensate for the major deficiencies of the ideal model is called here "reciprocal numerical mapping" of (the above) datasets. Each disclosed mapping rule furnishes the replacement of a fitting parameter by the relevant physical quantities including the variable exponent $\mathrm{p}^{*}$. As a whole, reciprocal mapping leads to the sought semi-ideal model, $\langle S I\rangle$.

The above numerical method is exceptionally simple and remarkably accurate; the major disadvantage lies in the fact that it only furnishes a special solution rather than a general one.

Before going into the details of the new evaluation technique, the collected experimental data will be presented and analyzed.

\section{Experimental and Numerical Results}

From the early functionality tests of the sensor, a lot of experimental findings already were at hand when this study was started. The key measurands of the sensor for the subsequent determination of velocity of fluid flow are the amplitude of a peak, $\Delta U\left(t_{\max }\right) \propto \Delta T\left(t_{\max }\right)$, and the related time of occurrence, $t_{\max }$. Those results on the latter two variables that were relevant to generate mapping rules between, e.g., the fitting and the ideal model parameters, $A^{*} \mapsto A, B^{*} \mapsto B$ and $\mathrm{p}^{*} \mapsto \mathrm{p}$, will be presented first.

Subsequently, the outcome of additional performance tests and virtual experiments of the heater will be described. For the latter task, the already mentioned three-dimensional finite element model for simulations was developed and experimentally verified. It provides a detailed insight into the transient and steady-state temperature response of the sensor. For instance, the experimental results of transient hot-wire (THW) runs on the sensor to identify the effective radius of its heater and to locate and assess potential heat losses could be confirmed by FEM experiments.

To simplify matters, all relevant experimental results are presented here by way of a typical set of sensor output voltages, i.e., the voltage drops of the RTDs T050, T100, T200, T300, and T400, in response to a 30- $\mu$ s long electrical square pulse. The quiescent gas inside the mini-channel of the sensor was $80 \mathrm{CH}_{4}-20 \mathrm{~N}_{2}$ at $23{ }^{\circ} \mathrm{C}$ (Fig. 5). The T050 voltage transient was chosen to help explaining the mapping procedure and to back up its outcome. Selected material properties of the above test medium are listed in Table 2. Figure 5 also presents the individual fitting curves according to Eq. 6. Figure 6 shows an enlarged view on the 
Table 2 Selected tabulated material properties of $80 \mathrm{CH}_{4}-20 \mathrm{~N}_{2}$ for room temperature $\left(23{ }^{\circ} \mathrm{C}\right)$ and atmospheric pressure (1013.25 mbar) [4]

\begin{tabular}{lllll}
\hline Gas & $\begin{array}{l}\text { Thermal conductivity } \\
{\left[\mathrm{W} \cdot \mathrm{m}^{-1} \cdot \mathrm{K}^{-1}\right]}\end{array}$ & $\begin{array}{l}\text { Thermal diffusivity } \\
{\left[\mathrm{m}^{2} \cdot \mathrm{s}^{-1}\right]}\end{array}$ & $\begin{array}{l}\text { Density } \\
{\left[\mathrm{kg} \cdot \mathrm{m}^{-3}\right]}\end{array}$ & Specific heat $\left[\mathrm{J} \cdot \mathrm{kg}^{-1} \cdot \mathrm{K}^{-1}\right]$ \\
\hline $\begin{array}{c}80 \mathrm{CH}_{4}- \\
20 \mathrm{~N}_{2}\end{array}$ & 0.027 & $21.55 \cdot 10^{-6}$ & 1.06 & 1189 \\
\hline
\end{tabular}

Table 3 Levenberg-Marquardt fitting parameters, Eq. 7, of the five output signals shown in Fig. 5

\begin{tabular}{lllllllll}
\hline RTD & $\begin{array}{l}\text { Distance to } \\
\text { heater }[\mu \mathrm{m}]\end{array}$ & $A^{*}$ & $B^{*}[\mathrm{~s}]$ & $\mathrm{p}^{*}$ & \multicolumn{1}{l}{$t_{\max }[\mathrm{s}]$} & $t_{\max }^{*}[\mathrm{~s}]$ & $t_{\max }^{+}[\mathrm{s}]$ & $\Delta U_{\max }[\mathrm{V}]$ \\
\hline T050 & 50 & $1.475 \times 10^{-7}$ & $4.72 \times 10^{-4}$ & 1.69 & $2.87 \times 10^{-4}$ & $2.79 \times 10^{-4}$ & $2.90 \times 10^{-5}$ & 0.028 \\
T100 & 100 & $1.788 \times 10^{-7}$ & $8.20 \times 10^{-4}$ & 1.68 & $4.92 \times 10^{-4}$ & $4.88 \times 10^{-4}$ & $1.16 \times 10^{-4}$ & 0.012 \\
T200 & 200 & $8.425 \times 10^{-8}$ & $1.72 \times 10^{-3}$ & 1.80 & $0.953 \times 10^{-3}$ & $0.956 \times 10^{-3}$ & $4.64 \times 10^{-4}$ & 0.004 \\
T300 & 300 & $7.947 \times 10^{-9}$ & $3.30 \times 10^{-3}$ & 2.20 & $1.497 \times 10^{-3}$ & $1.501 \times 10^{-3}$ & $1.04 \times 10^{-3}$ & 0.002 \\
T400 & 400 & $4.349 \times 10^{-10}$ & $5.81 \times 10^{-3}$ & 2.80 & $2.030 \times 10^{-3}$ & $2.080 \times 10^{-3}$ & $1.86 \times 10^{-3}$ & 0.001 \\
\hline
\end{tabular}

Also included are the observed points in time, $t_{\max }$, as well as the calculated points in time, $t_{\max }^{*}=B^{*} / \mathrm{p}^{*}$, (regression model) and $t_{\max }^{+}$(ideal model), respectively. The right most column contains the experimental peak amplitude, $\Delta U_{\max }$. The gas mixture is $80 \mathrm{CH}_{4}-20 \mathrm{~N}_{2}$

experimental and fitted data of the T400 thermometer to demonstrate the high fitting performance, even for noisy output signals.

The fitting parameters, $A^{*}, B^{*}$, and $\mathrm{p}^{*}$, of the above set of five curves, are listed in Table 3 along with the amplitudes of the related peaks, $\Delta U_{\max }$, and each point in time of the respective peaks from observation and calculations, respectively. The percentage differences between the experimental and the fitted values of the time of peak, $\left(t_{\max }^{*}-t_{\max }\right) / t_{\max }$, only vary between $-2.8 \%$ and $+2.4 \%$.

According to Table 3 , the difference in the predicted times of flight, $t_{\max }^{*}$ and $t_{\max }^{+}$, from the heater to the thermometer T050 amounts to $250 \mu \mathrm{s}$. For the successive RTDs, this latter time lag decreases with increasing distance from the heater.

\subsection{Temperature Signal}

In the ideal model, Eq. 1 with $\mathrm{p}=1$, the location- and time-dependent amplitude of the temperature transient, $\Delta T(t)$, depends on both ratios $A / t$ and $B / t$ where, for any given set of thermal quantities, $A$ is a constant and $B=B\left(x^{2}\right)$. In the regression model, the fitting parameters $A^{*}$ and $\mathrm{p}^{*}$ no longer are constant (Table 3 ). Obviously, the parameter, $A^{*}$, varies with the distance $x$ from the heater. However, it was not possible to find a reasonable functional relationship, $A^{*}=A^{*}(x)$, for this observation. The exponent $\mathrm{p}^{*}(x)$ can piecewise (T100 to T400) be fitted to a second-degree polynomial (Fig. 7). The variation of $B^{*}$ with $x^{2}$ at first sight appears to be almost linear. As is yet to be demonstrated, the latter correlation is erroneous because actually $B^{*}=B^{*}\left(x, \mathrm{p}^{*}\right)$. 


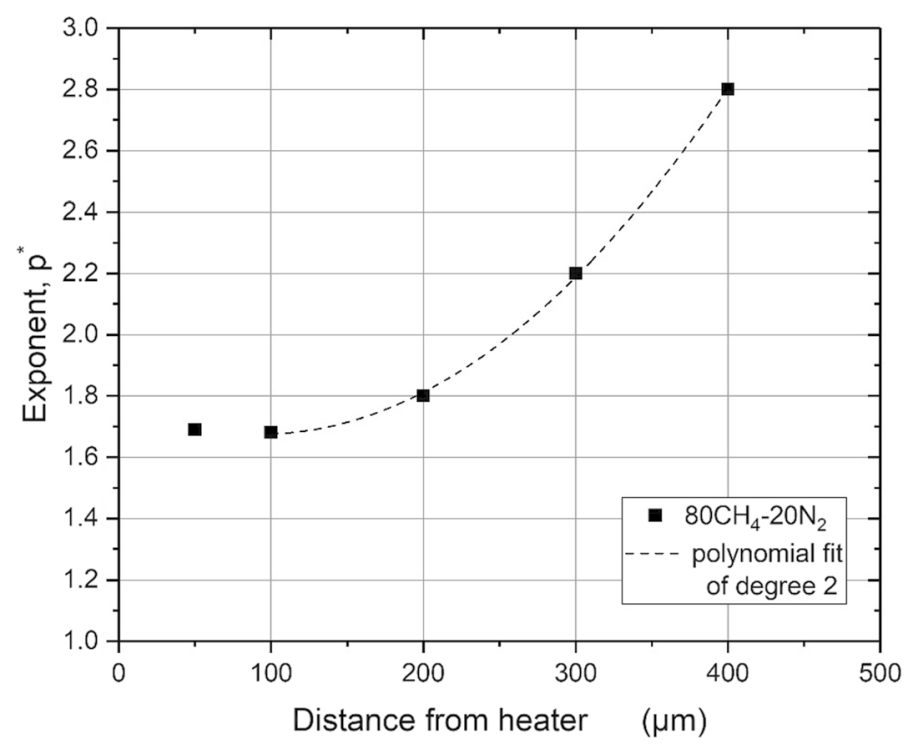

Fig. 7 Exponent $\mathrm{p}^{*}=\mathrm{p}^{*}(x)$ vs. distance from heater $x$. The polynomial fit is of $2^{\text {nd }}$ degree

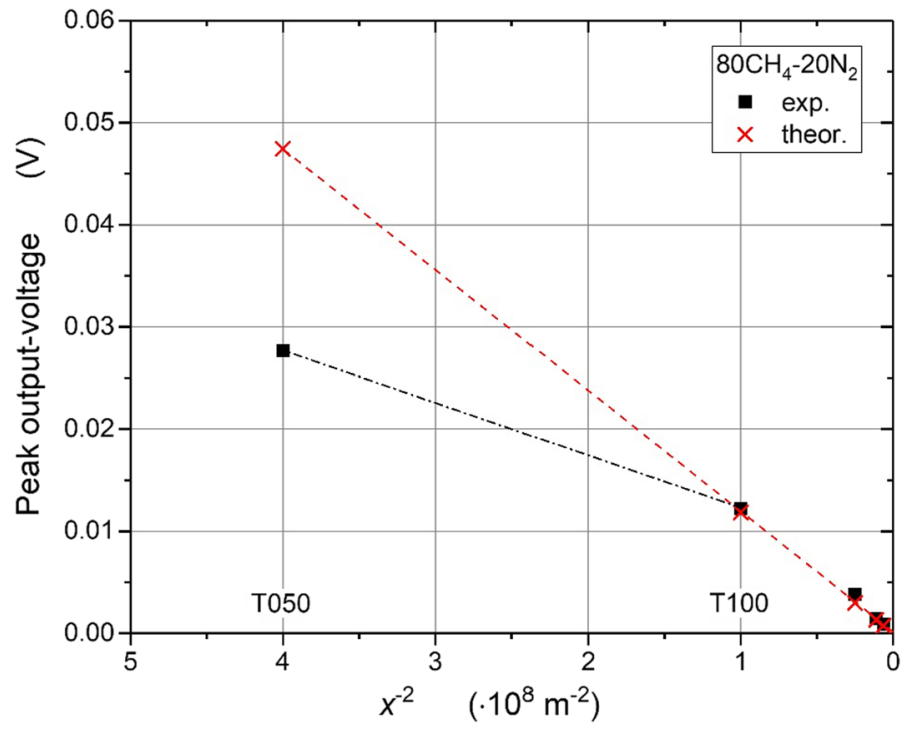

Fig. 8 Experimental output voltages, $\Delta U_{\max }\left(x^{-2}\right) \propto \Delta T_{\max }\left(x^{-2}\right)$, of the five peaks shown in Fig. 5 vs. reciprocal square distance from the heater. Theoretical values according to the ideal model

\subsubsection{Peak Amplitude}

In Fig. 8, the experimental peak output voltages, $\Delta U_{\max }$, of the five RTDs are plotted vs. $1 / x^{2}$ (cf. Eq. 5) together with their ideal model ("theor.") equivalents. 


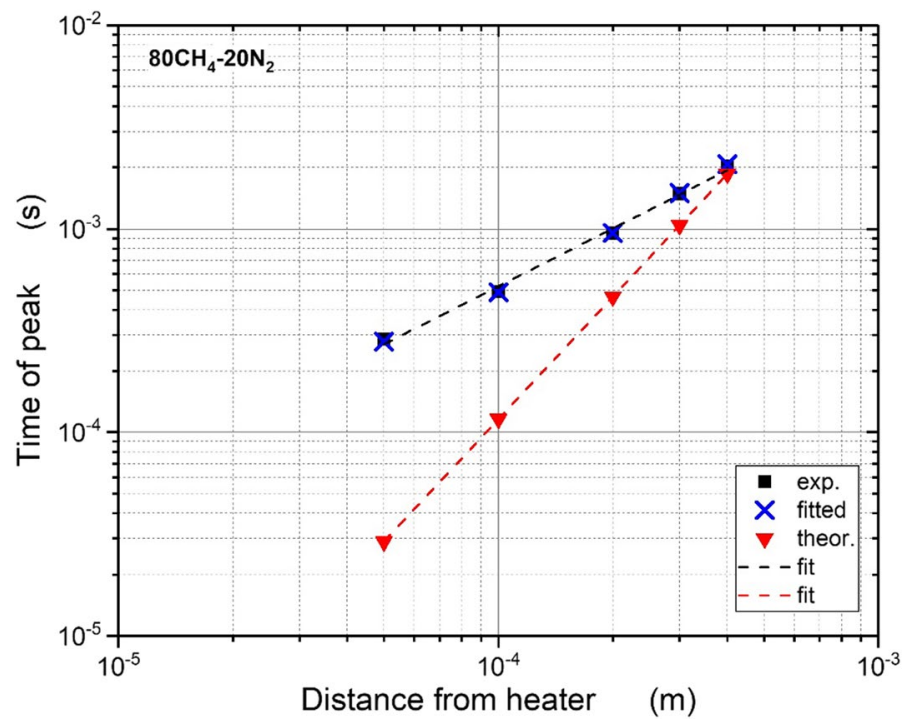

Fig. 9 Times of peaks of the five curves plotted in Fig. 5. (exp.: experimental data; fitted: fitted exp. data; theor.: ideal model data)

T100, T200, T300, and T400 yield data that are quite similar to the respective theoretical ones and, thus, can pretty well be quasi-linear fitted. The peak of the T050 signal behaves as an outlier in the regression dataset. The reason for this special role will be discussed in Sect. 6.2.

\subsection{Time of Peak}

The second key measurand of the sensor is the time of the occurrence of a peak. According to Eq. 4, in the ideal model $t_{\max }^{+} \propto x^{2}$. Consequently, the log-log plot in Fig. 9 shows the theoretical data as a quasi-linear fit of slope $m=2$. The experimental data (Table 3) can likewise be quasi-linear fitted. However, in contrast to the ideal model, the slope is $m=1$, rather than $m=2$, i.e., $t_{\max } \approx t_{\max }^{*} \propto x$.

\section{Semi-ideal Model I}

The ideal model of an instantaneous line heat source is that particular solution out of only a few closed-form analytical solutions of the heat conduction equation that comes as close as possible to the scenario of the PTF sensor. Nevertheless, this model markedly fails to predict the transient temperature response of the sensor (Fig. 2). However, in its parameterized form, the regression model, the observed signals are numerically predicted with astonishing accuracy (Figs. 5 and 6). However, the fitting parameters could not be related to the sought thermal parameters 


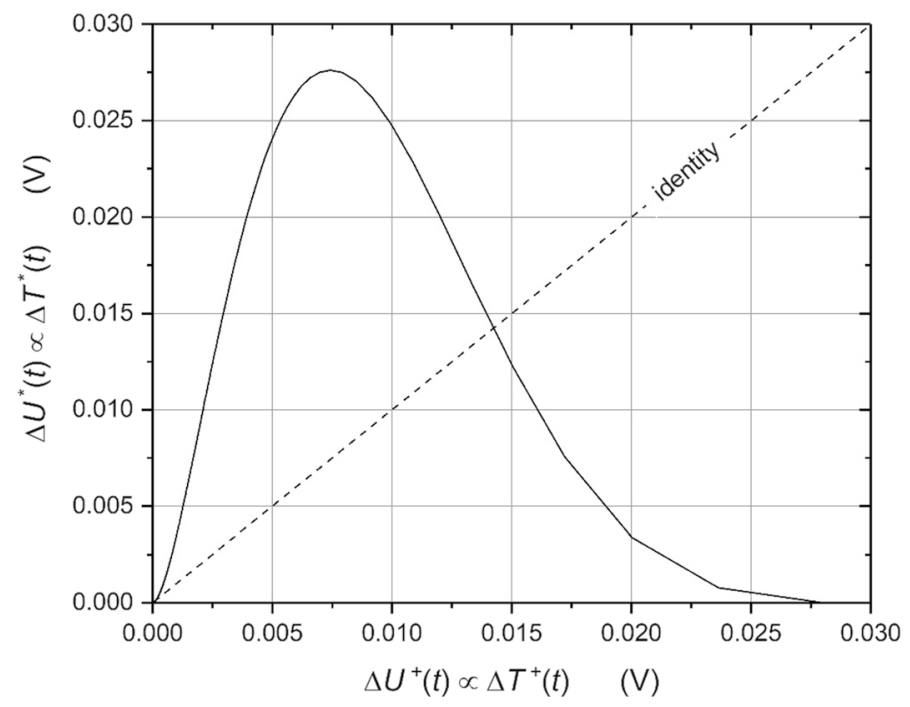

Fig. 10 One-to-one mapping from an ideal model set, $\Delta T^{+}(t)$, to a regression model set, $\Delta T^{*}(t)$, (see text). The dashed line indicates an identity of two sets

of the gas under test. Moreover, the regression model appears to be dimensionally inhomogeneous.

To resolve the above apparent dilemma, it was assumed that the ideal model is incomplete rather than inappropriate. If this latter hypothesis is realistic, then a mapping rule $f^{\prime}$ should exist that associates each element of an ideal model dataset, $\langle I\rangle$, with a unique element of the related regression model dataset, $\langle R\rangle$, or of the experimental dataset, $\langle U\rangle$. Since we are dealing here with numerical values, "element" means a real number out of a sequence of real numbers that are indexed by natural numbers in discrete-time order. For instance, the series of output voltages of a sensor run sampled from a continuous time signal at 501 discrete points in time is termed an experimental dataset. The linked regression dataset, $\langle R\rangle$, is generated by fitting the experimental data according to Eq. 7. And finally, the related ideal dataset, $\langle I\rangle$, is calculated from the experimental input data according to Eq. 2. For a mapping from, e.g., $\langle I\rangle$ to $\langle R\rangle(f:\langle I\rangle \mapsto\langle R\rangle)(1)\langle I\rangle$ and $\langle R\rangle$ should be non-empty and (2) each element of $\langle I\rangle$ should have an image in $\langle R\rangle$. Due to its mathematical complexity, here, each mapping is carried out numerically rather than analytically. Hence, the results obtained may not readily be generalized, i.e., they cannot be a "clear-cut answer" to the above hypothesis.

Figure 10 presents the injective mapping of the ideal T050 voltage signal (cf. Fig. 5) to the related fitted experimental data. The underlying thermal parameters of both signals are identical. Prior to the mapping, the ideal dataset was normalized to the maximum of the fitted dataset. By definition, both sets are (naturally) nonempty and each element (voltage), $\Delta U^{+} \in\langle I\rangle$, of the ideal dataset has an image, $\Delta U^{*} \in\langle R\rangle$, in the regression dataset. The plot of the function, $f:\langle I\rangle \mapsto\langle R\rangle$, exists and may be considered continuous and differentiable, i.e., principally, one ideal 
model set can be one-to-one mapped into one regression/experimental model set. The dashed line inside the above diagram illustrates an identity map of both sets, $f(x)=x$.

So far, no elementary function could be guessed to analytically express the entire asymmetric peak curve presented in Fig. 10. Therefore, feasible mappings were performed termwise, i.e., (1) for the time of a peak and (2) for the peak amplitude as will be shown in the next section. The ideal datasets of the composite variable, $A(=$ const. $),\langle A\rangle$, and the exponent $\mathrm{p}(=1),\langle\mathrm{p}\rangle$, respectively, may not be mapped since both only have one member.

\subsection{Termwise Mapping}

The key-information of a PTF-signal to determine the velocity of flow is contained in the time and amplitude of a peak:

$$
\begin{gathered}
\text { (1) } t_{\text {max }}^{+} \mapsto t_{\max }^{*} \Rightarrow \frac{B}{\mathrm{p}}=\frac{r^{2}}{4 a} \mapsto \frac{B^{*}}{\mathrm{p}^{*}} \text { and } \\
\text { (2) } \Delta T_{\max }^{+} \mapsto \Delta T_{\max }^{*} \Rightarrow \frac{A}{t_{\text {max }}^{+} \mathrm{e}}=\frac{H}{4 \pi L \lambda t_{\max }^{+} \mathrm{e}} \mapsto \frac{A^{*}}{\left(t_{\max }^{*} \mathrm{e}\right)^{\mathrm{p}^{*}}},
\end{gathered}
$$

respectively.

\subsubsection{Time of Peak}

Figure 11 presents the first one of the above two mappings, $B^{*} / \mathrm{p}^{*}=f(B / \mathrm{p})$. This $\log -\log$ graph reveals a quasi-linear relationship between both sets. A fit furnishes the slope $m=0.483 \approx 0.5$ and the intercept $\mathrm{n}=-1.385$. Hence, the mapping rule reads $B^{*} / \mathrm{p}^{*}=n B^{m}=0.0412 B^{0.483} \approx 0.0412 \sqrt{B}$. When plotted against $\sqrt{B}$ (Fig. 12), one gets the expected quasi-linear curve of slope $q=0.04771 \mathrm{~s}^{1 / 2}$ and intercept, $n=-2 \cdot 10^{-5} \mathrm{~s} \approx 0$, i.e., the intercept vanishes. The fitting parameter $B^{*}$ reads:

$$
B^{*}=q \mathrm{p}^{*} \sqrt{B}=\frac{q \mathrm{p}^{*} x}{2 \sqrt{a}}
$$

The dimensional unit of this result is $\left[B^{*}\right]=\mathrm{s}$. Recalculated in terms of the time exponent, one gets $\mathrm{p}^{*}=B^{*} /(q \sqrt{B})$. The parameter $q$ has to be treated as a sensor constant.

\subsubsection{Amplitude of Peak}

The diagram Fig. 13 visualizes the second mapping, $A^{*} /\left(t_{\max }^{*} \mathrm{e}\right)^{\mathrm{p}^{*}}=f\left(A /\left(t_{\max }^{+} \mathrm{e}\right)\right)$. Here, the T050 data point again (cf. Fig. 8) appears as an outlier (cf. Sect. 6.2). 


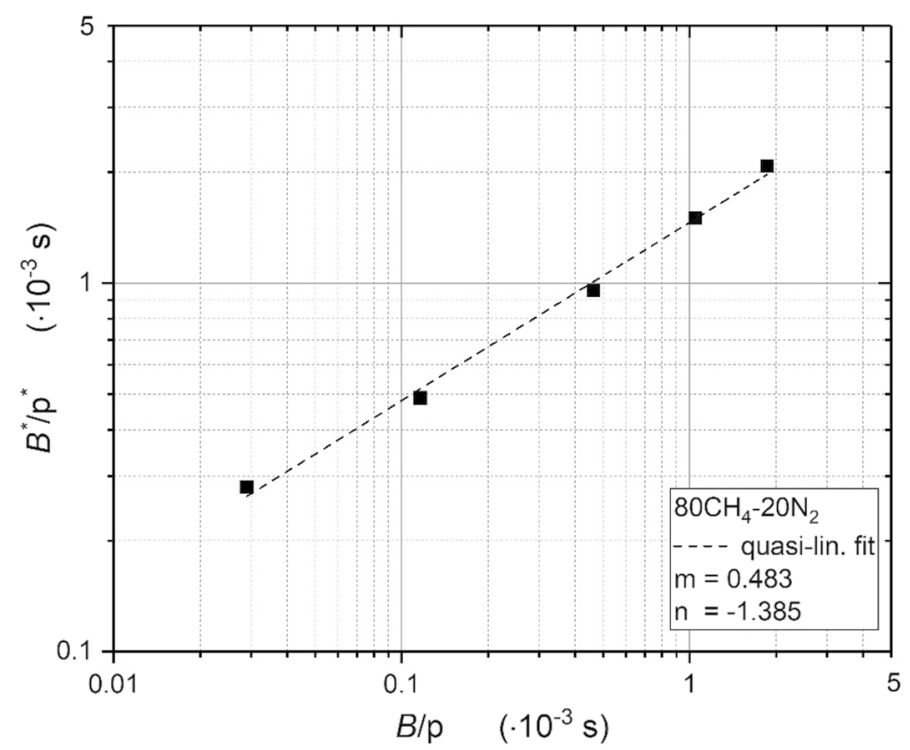

Fig. 11 Log-log plot of experimental data $B^{*} / \mathrm{p}^{*}$ vs. ideal model composite variable $B / \mathrm{p}(\mathrm{p}=1)$ (cf. Fig. 9)

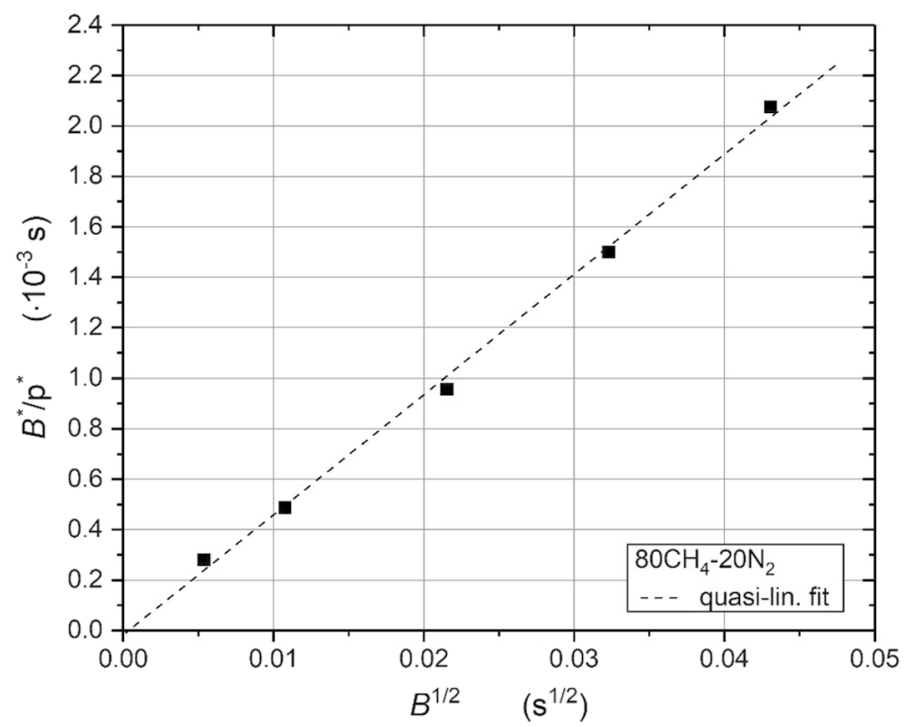

Fig. 12 Fitting parameter $B^{*} / \mathrm{p}^{*}$ vs. square root of ideal model composite variable $\mathrm{B} / \mathrm{p}(\mathrm{p}=1)$

The linear fit to the four data T100, T200, T300, and T400 furnishes the slope $m(\mathrm{~g} 2)=\mu_{2}=0.539 \mathrm{~s}^{1-\mathrm{p}^{*}}$; whereas the line segment from the origin to T050 is of slope $m(\mathrm{~g} 1)=\mu_{1}=0.310 \mathrm{~s}^{1-\mathrm{p}^{*}}$. The two intercepts, $n_{\mathrm{i}}$, vanish. The general mapping rule, thus, reads $A^{*} /\left(t_{\max }^{*} \mathrm{e}\right)^{\mathrm{p}^{*}}=\mu\left(A /\left(t_{\max }^{*} \mathrm{e}\right)\right) \Leftrightarrow \Delta T_{\max }^{*}=\mu \Delta T_{\text {max }}^{+}$. From 


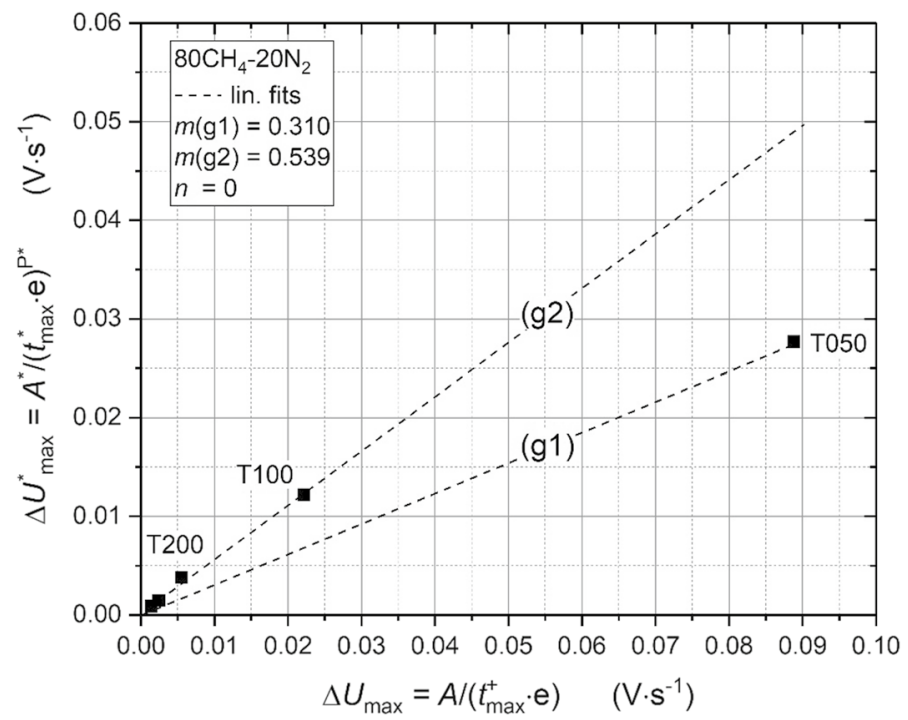

Fig. 13 Fitting variable $A^{*} /\left(t_{\max }^{\mathrm{p}^{*}} \mathrm{e}^{\mathrm{p}^{*}}\right)$ vs. ideal model composite variable $A / t_{\max }(\mathrm{cf}$. Fig. 8$)$. The two dashed lines (gi) are linear fits from the origin to the point T050 (g1) and from T100 to T400 (g2), respectively

$\Delta T_{\max }^{*} \leq \Delta T_{\max }^{+}$, one gets $0 \leq \mu \leq 1$ for this sensor constant. The above mapping also yields the dimensional unit of the latter coefficient, $[\mu]=\mathrm{s}^{1-\mathrm{p}^{*}}$. Finally,

$$
A^{*}\left(\mathrm{p}^{*}(x)\right)=\frac{\mu}{\left(\mathrm{p}^{*}\right)^{\mathrm{p}^{*}} \mathrm{e}^{\left(1-\mathrm{p}^{*}\right)}} \frac{\left(B^{*}\right)^{\mathrm{P}^{*}}}{B} A=\sigma\left(\mathrm{p}^{*}(x)\right) A \text { with } \sigma(p(x))=\frac{\mu}{\left(\mathrm{p}^{*}\right)^{\mathrm{p}^{*}} \mathrm{e}^{\left(1-\mathrm{p}^{*}\right)}} \frac{\left(B^{*}\right)^{\mathrm{p}^{*}}}{B} .
$$

The variation of $\sigma$ with $\mathrm{p}^{*}$ indicates that this parameter depends on the actual waveform of the enthalpy pulse liberated by a heater of non-vanishing heat capacity (cf. Sect. 6). In the trivial case of identity ( $A^{*}=A=$ const.), the following conditions are valid: $\mathrm{p}^{*}=1, \mu \equiv 1$ and $B^{*} \equiv B$. The coefficient $\sigma$ is of dimensional unit $[\sigma]=\mathrm{s}^{1-\mathrm{p}^{*}}$.

\subsection{Input Enthalpy}

Each curve fitting of an output signal of the PTF sensor furnished a time exponent $\mathrm{p}^{*}=\mathrm{p}^{*}(x)>1.5$ whereas the relevant ideal model only covers $\mathrm{p} \equiv 1$. Consequently, from the regression model condition $\left[A^{*} / t^{\mathrm{p}^{*}}\right] \stackrel{\mathrm{K}}{=} \mathrm{K}$, the dimensional unit of the proportionality factor $\mu$ of the above (dimensional) mapping is $\mathrm{s}^{1-\mathrm{p}^{*}}$ instead of 1 . It is expected that actually $A^{*}(x) / t^{\mathrm{p}^{*}}=A^{*}(x) \cdot t^{1-\mathrm{p}^{*}} / t$, so that $A^{*}(x) \propto \mu H_{0} t^{\left(1-\mathrm{p}^{*}(x)\right)}$ instead of $A \propto H_{0}$.

The physical scenario behind the assumed variation of the input enthalpy with time including the relevance of the time exponent is discussed in Sect. 6.1. 


\section{Semi-ideal Model Equations}

Compiling the above partial results, one finally gets the semi-ideal model, $\langle S I\rangle$ :

$$
\Delta T(x, t)=\frac{1}{4 \pi L \lambda t} \exp \left(\frac{-x^{2}}{4 a t}\right) \cdot H(x) \sigma t^{1-\mathrm{p}^{*}} \exp \left(\frac{x^{2}}{4 a t}-\frac{q \mathrm{p}^{*} x}{2 \sqrt{a} t}\right)
$$

or, recalculated,

$$
\Delta T(x, t)=\frac{H(x) \sigma t^{1-\mathrm{p}^{*}}}{4 \pi \lambda L t} \exp \left(\frac{-q \mathrm{p}^{*} x}{2 \sqrt{a} t}\right) .
$$

When compared with the ideal model relation, Eq. 1, Eq. 14 contains two "disturbance terms," (1) $H(x, t)$ instead of $H=$ const. and $\exp \left[x^{2} /(4 a t)-q^{*} \mathrm{p}^{*} x /(2 \sqrt{a} t)\right]$ instead of $\exp \left(x^{2} /(4 a t)\right)$ (cf. Sect. 6).

The temperature maximum,

$$
\Delta T_{\max }=\frac{H\left(x, t_{\max }\right) \sqrt{a}}{2 \pi L \lambda q x}
$$

occurs at time

$$
t_{\max }=\frac{q x}{2 \sqrt{a}}
$$

It is worth noting here that $t_{\max }$ does not depend on the exponent $\mathrm{p}^{*}$. In principle, the thermal diffusivity can be determined by the PTF sensor according to the working equation

$$
a=\left(\frac{q x}{2 t_{\max }}\right)^{2}
$$

Figure 14 presents the standard percentage departure plot of the T050 experimental data from the calculated semi-ideal model data. The very small but rapidly increasing deviations at short times are due to the fact that the ideal and the semiideal models deal with an infinite pulse of enthalpy at $t \rightarrow 0$ whereas for the regression model the enthalpy pulse is finite. Figure 15 shows a mapping of the generated dataset $\langle S I\rangle$ to the experimental dataset $\langle U\rangle$. While the falling edge of the T050 pulse function closely approximates an identity relation (dotted red line), the rising edge (marked by arrows) somewhat deviates. The latter departure can also be seen in Fig. 14 for small times. 


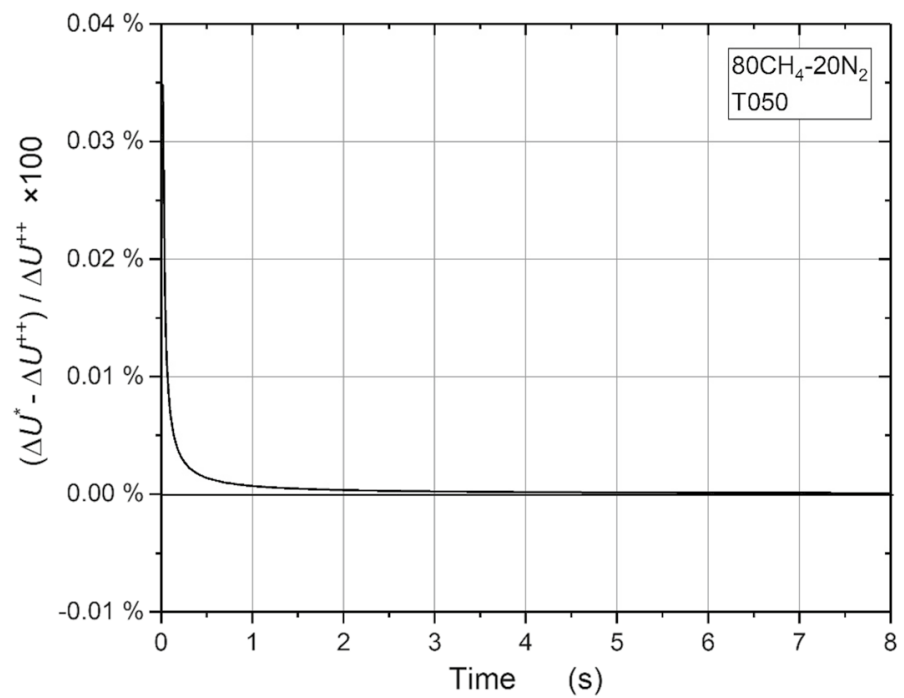

Fig. 14 Percentage departure of the T050 fitted experimental data, $\Delta U^{*}$, from the calculated data according to the semi-ideal model, $\Delta U^{++}$

\section{Semi-ideal Model II/Physical Interpretation}

The semi-ideal model has to deal with at least four physical peculiarities: (1) the unusual time exponent, $\mathrm{p}^{*}=\mathrm{p}^{*}(x)>1.5$, instead of $\mathrm{p}=1=$ const. (Fig. 7), (2) the "T050 outlier" (Figs. 8 and 13), (3) the unusual argument of the exp-function, (Eq. 15), and (4) the path dependence of $A^{*}$ (Table 3 ).

To understand the above heat propagation phenomena during a test sequence of the sensor, the scenery may be confined to the area of the active components $\left(2000 \times(500+50) \times 0.5 \mu \mathrm{m}^{3}\right)$ of the sensor: its heater and $5+1$ thermometers (Fig. 1). The complete scenario can be considered as one-dimensional propagation of a heat pulse from the source, passing four thermometers, and finally reaching the fifth thermometer within less than $10 \mathrm{~ms}$.

Explanations to the above peculiarities will be given in Sects. 6.2, 6.3.1, and 6.3.2, printed in cursive characters.

\subsection{Semi-ideal Heater and Thermometers}

Due to its non-vanishing thermal mass, the heater is capable of storing heat. The actual amount of stored heat is proportional to the transient temperature difference between the heater and the surrounding gas. Since the thermometers are identical in construction, the latter components are likewise able to store heat proportional to their individual temperature differences to the gas.

In the ideal case of a line heat source at $r=0$ that is excited by a Dirac pulse of enthalpy, $H_{0}$, the excess temperature drops from its initial value, $\Delta T(r=0, t=0)$ 


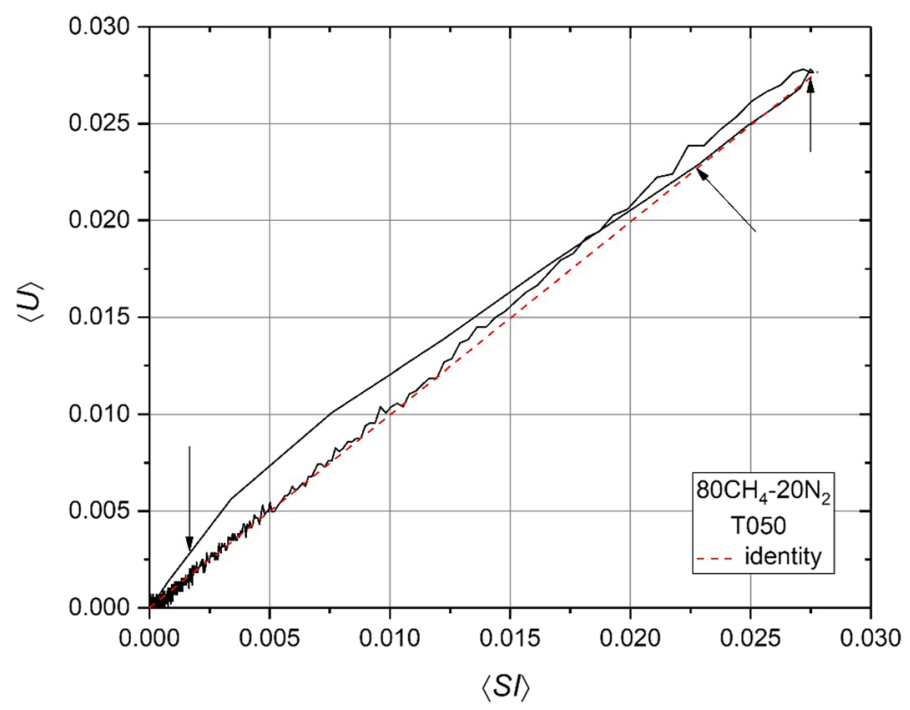

Fig. 15 Mapping from the semi-ideal model set, $\langle S I\rangle$, to the experimental set, $\langle U\rangle$. The segment of the curve marked by three arrows is a slight "mismapping" due to the departures at short times (see Fig. 14). Identity means: $f(x)=x$

to $\Delta T=0$ proportional to $H_{0} / t$. According to the regression model, the excess temperature instead drops with $H_{0} / t^{\mathrm{p}^{*}(x)}$ where $\mathrm{p}^{*}(x) \approx c_{2}(x-100)^{2}+c_{0}$ (Table 3) for the T100, T200, T300, and T400 data points. ${ }^{3}$ Within the relevant period, $t \leq 1 \mathrm{~s}$, the latter decline is less steep than in the ideal case. This means that the larger the time exponent, the less steep the temperature drop per time unit and, consequently, the less steep the drop per time unit, the larger the heat capacity of the heater and the thermometers that one after the other take part in the conduction of heat from the source to the ambient. All in all, $\mathrm{p}^{*}$ is an indirect measure of the combined heat storing capabilities of heater and thermometers that accumulate during a run.

To simplify matters, the heater is considered as a line heat source that is surrounded by a solid cylinder of radius $\tilde{r}=3 \mu \mathrm{m}$. The thermal properties of this virtual cylinder are its virtual thermal conductivity, $\tilde{\lambda}$, and virtual thermal diffusivity, $\tilde{a}=\tilde{\lambda} /\left(\tilde{\rho} \tilde{c}_{p}\right)$, where $\left(\tilde{\rho} \tilde{c}_{p}\right)$ denotes the virtual volumetric-specific heat. Likewise, each RTD is considered as a "line thermometer" surrounded by a virtual cylinder of $r=\tilde{r}\left(\tilde{r}=\sqrt{\tilde{x}^{2}+\tilde{y}^{2}}\right), \tilde{\lambda}$ and $\tilde{a}$. Any delay in pulse propagation due to the storing and releasing of heat by the heater and/or a thermometer can be considered by means of the related virtual thermal diffusivity. The above numerical value of the radius of the virtual cylinder will be justified below (Sect. 6.3.1).

So far, the spacing between two adjacent microbridges was specified by the distance of their longitudinal axes. Considering the virtual cylinders, the

\footnotetext{
3 Again, the point T050 is an outlier.
} 
gas-domain gaps between two microbridges are $\Delta x=50 \mu \mathrm{m}-2 \cdot 3 \mu \mathrm{m}=44 \mu \mathrm{m}$ and $\Delta x=94 \mu \mathrm{m}$, respectively.

\subsection{Time Sequence of a Run}

A run is started by applying an electrical square pulse of $30 \mu$ s to the heater. In the semiideal model this means that, for a period of $30 \mu \mathrm{s}$, the line source continuously liberates a predefined constant rate of heat flow into the surrounding virtual cylinder. Here, the heat pulse is conducted through the gas domain within the period $t_{\max }^{(1)}=\tilde{x}^{2} /(4 a)$. The first gas-filled gaps on either side of the heater, each of width $\Delta x_{050}= \pm 44 \mu \mathrm{m}$, are traversed within $t_{\max }^{(2)}=x_{050}^{2} /\left(4 a_{\text {Gas }}\right)$. Before the pulse simultaneously reaches the line thermometers T050 $(+44 \mu \mathrm{m})$ and TL050 $(-44 \mu \mathrm{m})$, their virtual cylinders have to be passed halfway, $t_{\max }^{(3)}=x^{2} /(4 a)$. The line thermometers monitor the temperature history without any delay. Both their virtual cylinders equally share the actual amount of available heat.

(1) Since all other RTDs except the T050 do not have any counterpart on the other side of the heater, the T050 temperature maximum appears as outlier in Figs. 8 and 13. After $t_{\max }^{(4)}=x^{2} /(4 a)$, the heat pulse travels through the $94 \mu \mathrm{m}$ width gas domain to T100 and its virtual cylinder and so on.

\subsection{Series Conduction of Heat and Storing}

On its way from the line source to the last line thermometer T500, the heat pulse six times either diffuses through a virtual cylinder (halfway or entirely) or through the gas domain. Both pairs of the relevant thermal transport properties $\lambda_{\text {Gas }}$ and $\tilde{\lambda}_{C}$ on the one hand as well as $a_{G a s}$ and $\tilde{a}_{C}$ on the other are alternating several times whereby $\lambda_{\text {Gas }}<<\tilde{\lambda}_{C}$ and $a_{\text {Gas }}>>\tilde{a}_{C}$.

In the relevant literature, a pipe composed of coaxial cylinders of materials each of different thickness and distinct thermal transport properties is known well as "composite cylindrical wall." For such an arrangement, overall transport properties can be defined, e.g., an overall thermal conductivity, $\lambda_{0}$, and an overall thermal diffusivity, $a_{0}$. Both latter quantities depend on all individual transport properties of the cylindrical layers and all respective thicknesses. With each additional layer, the overall transport properties change. Due to the latter path dependence, these composite quantities are no longer material constants.

Presumed, the transport of heat discussed here may be almost entirely restricted to the above-defined active area of the sensor then the deviations of the measured times of peak from the ideal model ones (cf. Fig. 9) can be understood as a result of the composite-wall structure of the relevant heat path. The experimental results described below seem to confirm this hypothesis.

\subsection{Overall Thermal Diffusivity}

For a measurement of the overall thermal diffusivity of the active heat path of a gasfilled PTF sensor, $a_{0}=a_{0}\left(\tilde{a}_{C}, a_{G}, \tilde{r}_{C}, r_{G}\right)$, the sensor itself offers at least two distinct 


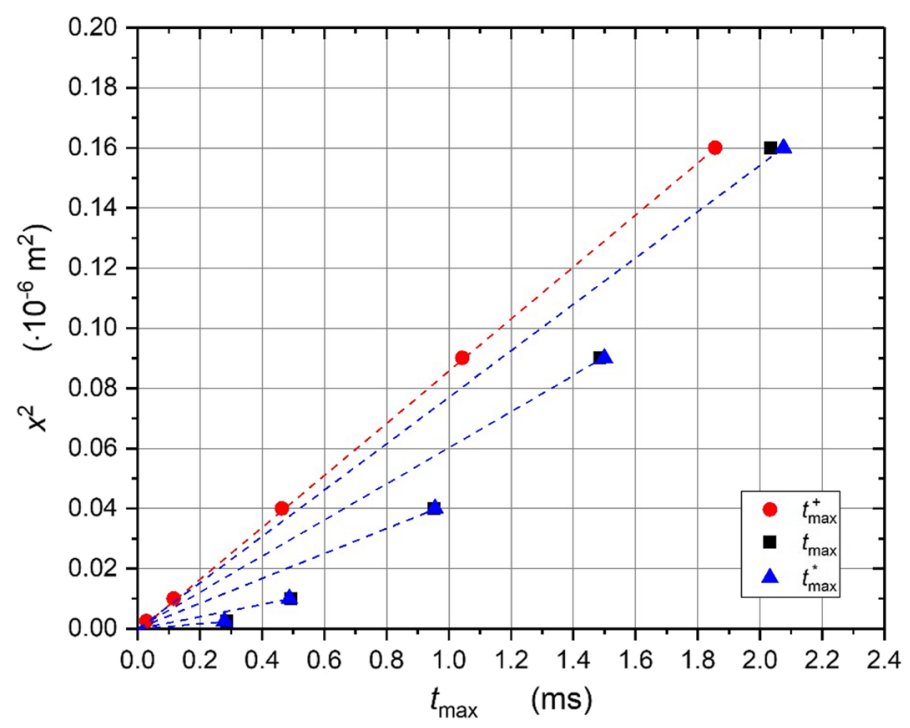

Fig. 16 Squared distance, $x^{2}$, vs. time of a peak for exp., $t_{\max }$, and theor. data calculated from the ideal, $t_{\max }^{+}$, and the regression models, $t_{\max }^{*}$. The slopes of the fitted lines are proportional to the local overall thermal diffusivities, $a_{0}, a_{0}^{+}$and $a_{0}^{*}$

techniques, the pulse mode of operation (PTF), discussed here so far (cf. Fig. 5) and the transient hot-wire (THW) method. The latter technique has already been multifariously presented and discussed in great detail [3, 5-10]. Therefore, here, only a brief insight is required.

The above first technique furnishes a "squared-path"-time diagram where the squared distance of the heat source to a temperature station, $x^{2}$, is plotted vs. the relevant time-of-travel for a peak. Figure 16 presents "squared-path"-time curves, $x^{2}=4 a t_{\max }=m t_{\max }$, for the three available distinct times of peak, $t_{\max }, t_{\max }^{+}$, and $t_{\max }^{*}$ (cf. Fig. 9). In the diagram, each single point in time is taken as one end point of a line segment whereas the other end point is the origin. From the slope, $m=4 a$, of every line segment, each local overall thermal diffusivity, $a_{0}\left(x^{2}, t_{\max }^{()}\right)$ can be calculated. In the ideal model $\left(t_{\max }^{+}\right)$, it is self-evident that all single slopes are identical. In the regression model $\left(t_{\text {max }}^{*}\right)$, however, the single slopes are distinct from each other, so are the specific local overall thermal diffusivities.

Compared with the PTF mode, the THW technique applied here, only requires a constant voltage step pulse of some $40 \mathrm{~ms}$ in place of the short PTF pulse of $30 \mu \mathrm{s}$. During a THW run, here, not only the transient temperature of the linear heat source is monitored but also those transients of all RTDs. The latter output voltages are depicted in Fig. 17 vs. the natural logarithm of time, $\Delta U(\ln (t))$. The quasi-linear interval of each curve is indicated by a fitted line segment. For the slope $m$ of a line segment, $m=\Phi /(4 \pi L \lambda)$ holds, for the intercept, $n=m \ln \left(4 a /\left(C r^{2}\right)\right)$. Here, the constant rate of liberated heat flow is denoted $\Phi$ 


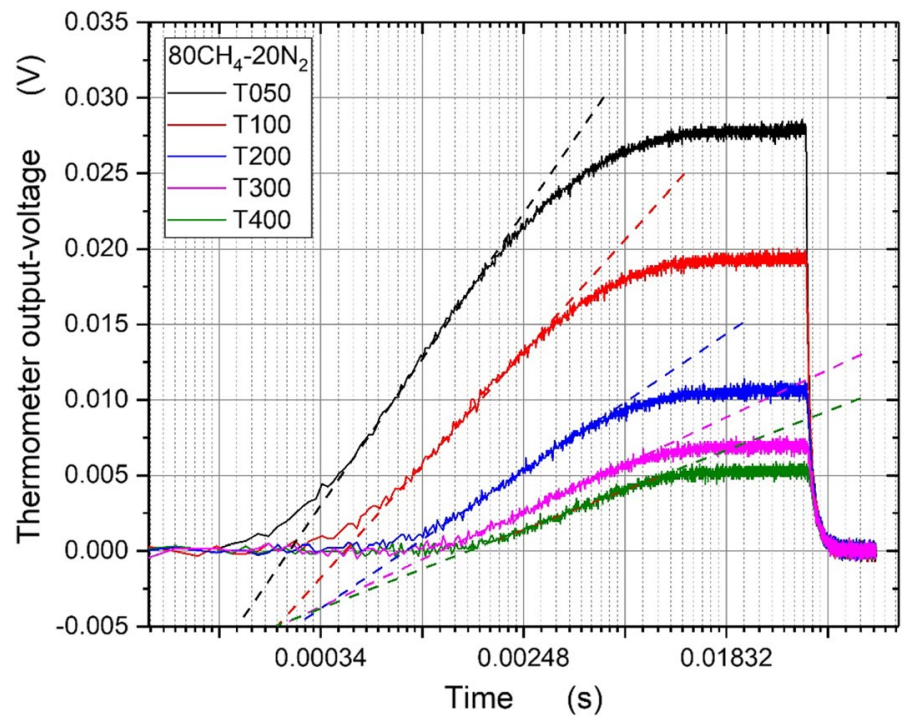

Fig. 17 RTD output signals of the indicated five thermometers Txxx vs. In $(t)$ during a transient hot-wire experiment on $80 \mathrm{CH}_{4}-20 \mathrm{~N}_{2}$ using the PTF sensor

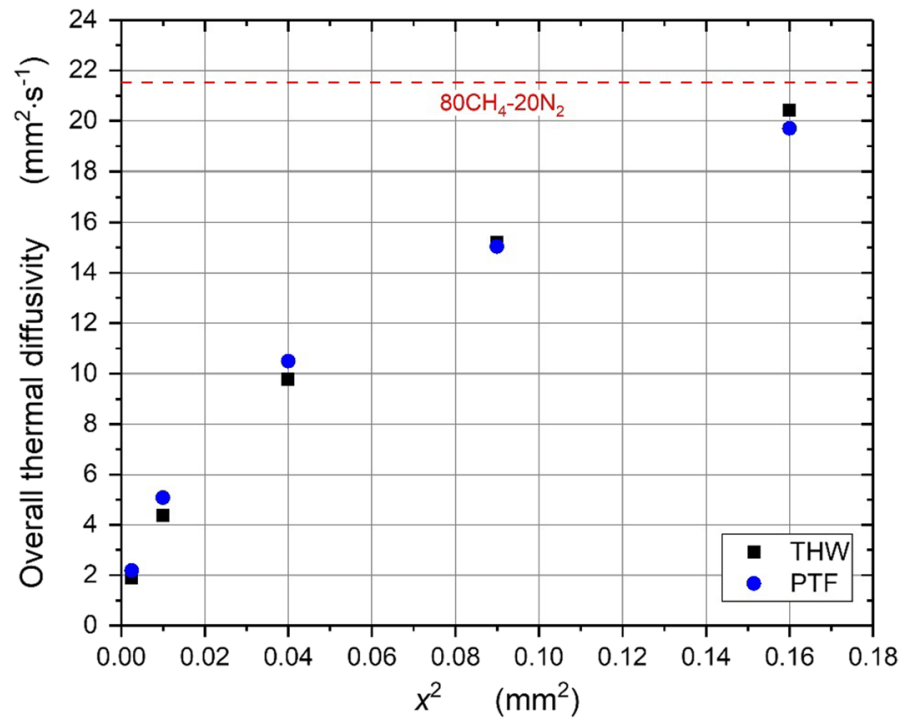

Fig. 18 Local overall thermal diffusivity, $a_{0}$, for the gas mixture $80 \mathrm{CH}_{4}-20 \mathrm{~N}_{2}$ vs. squared distance from the heater, $x^{2}$, for all five RTDs. For comparison purposes, the tabulated thermal diffusivity, $a$ of the gas is also plotted [4]

and $C \equiv \exp (\gamma)$ (Euler's constant: $\gamma$ ). Each slope furnishes the local (overall) thermal conductivity; from each slope and the associated intercept, the local (overall) thermal diffusivity follows. 


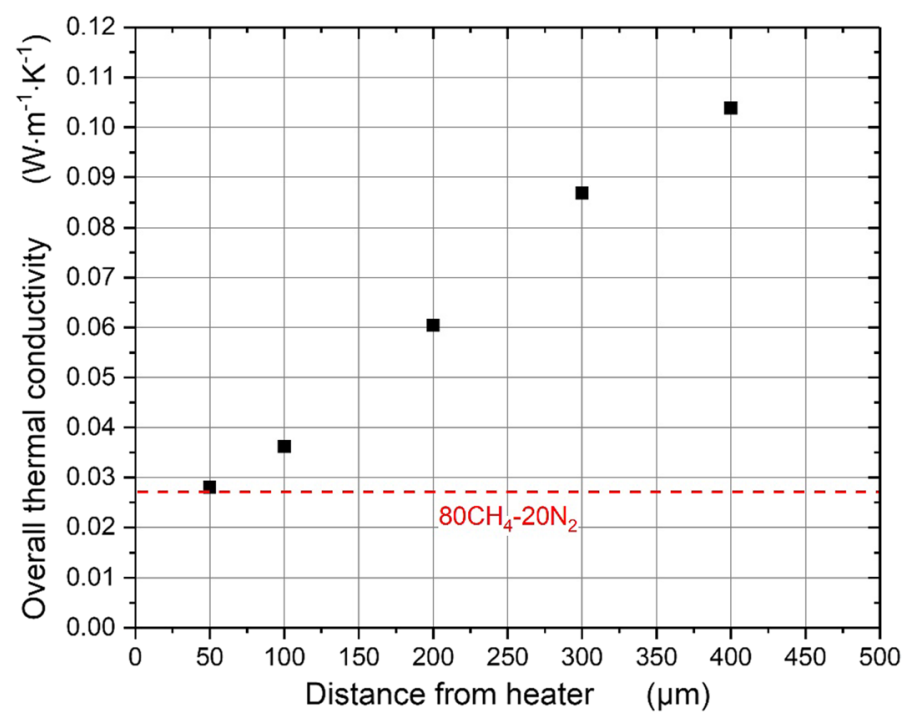

Fig. 19 Local overall thermal conductivities, $\lambda_{0}$, for the gas mixture $80 \mathrm{CH}_{4}-20 \mathrm{~N}_{2}$ vs. distance from the heater for all five RTDs. For comparison purposes, the tabulated thermal conductivity of the gas is also plotted [4]

In Fig. 18, all measured local overall thermal diffusivities, $a_{0}\left(x^{2}\right)$, are plotted vs. $x^{2}$. For comparison purposes, the tabulated thermal diffusivity of $80 \mathrm{CH}_{4}-20 \mathrm{~N}_{2}$ (Table 2: $a=21.55 \mathrm{~mm}^{2} \cdot \mathrm{s}^{-1}$ ) is given too.

Obviously, both procedural different measurement results agree very well if in the above relation for the intercept of the THW line segment the radius of the heat source is set to $\tilde{r}=3 \mu \mathrm{m}$. The overall thermal diffusivity of the active heat path of the gas-filled sensor increases in a way that the curve $a_{0}\left(x^{2}\right)$ gets closer and closer to $a^{+}=$const..

So far, there are only preliminary assessments of the measurement uncertainties of both the above sensor methods. It is estimated that the THW standard uncertainty is $10 \%$ for thermal diffusivity and $5 \%$ for thermal conductivity. The PTF standard uncertainty of thermal diffusivity primarily depends on the measurements of a period in time, $t_{\max }$, and of a distance, $x$, respectively. Therefore, the uncertainty is estimated to be better than $5 \%$ (cf., e.g., [11]).

\subsection{Overall Thermal Conductivity}

Figure 19 presents the experimental results of the THW tests on the local overall thermal conductivity, $\lambda_{0}=\lambda_{0}\left(\tilde{\lambda}_{C}, \lambda_{G}, \tilde{r}_{C}, r_{G}\right)$, vs. distance from the heater. Between $x=3 \mu \mathrm{m}$ (right outer edge of the heater microbeam) and $x=47 \mu \mathrm{m}$ (left outer edge of the T050 microbeam), the measured thermal conductivity of the gas mixture is almost equal $(-2 \%)$ to the tabulated value (Table 2). Between $x=47 \mu \mathrm{m}$ 
and $x=53 \mu \mathrm{m}$ (within T050), the intrinsic thermal conductivity of the RTD is much greater than that of the gas domain so that the overall thermal conductivity significantly increases. Then, inside the gas-domain again, the overall thermal conductivity decreases to $\lambda_{0}=0.036 \mathrm{~W} \cdot \mathrm{m}^{-1} \cdot \mathrm{K}^{-1}$ when reaching the next outer edge of the next RTD and so on.

With each passing of the heat through a virtual cylinder of a thermometer, the local overall thermal conductivity increases correspondingly while with each passing through the gas, the local overall thermal conductivity decreases again.

\subsection{Alternative Semi-ideal Model Equation}

The composite-wall approach is able to explain the above-mentioned peculiarities: (2) the unusual argument of the exp-function, (Eq. 15), and (3) the path dependence of $A^{*}$ (Table 3 ).

(2) The mapping procedure furnished the non-dimensional ratio $q \mathrm{p}^{*} x /(2 \sqrt{a})$ in place of the non-dimensional ratio $r^{2} /(4 a)$ of the ideal model. The compositewall approach instead yields the ratio.

$x^{2} / 4 a_{0}\left(x^{2}\right)$.The latter ratio is equal to the above semi-ideal term.

(3) The mapping procedure furnished the prefactor, $H(x) \sigma t^{1-\mathrm{p}^{*}} /(4 \pi \lambda L t)$, of the semi-ideal model. The composite wall instead deals with a local, path-dependent overall thermal conductivity, $H t^{1-\mathrm{p}^{*}} /\left(4 \pi \lambda_{0}(x) L t\right)$. Again, the results of mapping and composite-wall approach are equal.

The alternative semi-ideal model equation reads:

$$
\Delta T(x, t)=\frac{H t^{1-\mathrm{p}^{*}}}{4 \pi \lambda_{0}(x) L t} \exp \left(\frac{-x^{2}}{4 a_{0}\left(x^{2}\right) t}\right) .
$$

Finally, the sought working equation for the pulsed thermal flow (PTF) sensor can be formulated as follows:

$$
\Delta T(x, t)=\frac{H(x) \sigma t^{1-\mathrm{p}^{*}}}{4 \pi \lambda L t} \exp \left(\frac{-q \mathrm{p}^{*}(x-v t)}{2 \sqrt{a} t}\right)
$$

where the velocity of flow of the gas under test is denoted $v$.

\section{Conclusions}

The most important result of the presented study lies in the fact that the transient temperature output signals of the PTF sensor can be predicted at a reasonable degree of accuracy by the ideal model of an instantaneous line heat source when only minor adjustments are made. In order to finally come to this conclusion, the thermal transmission chain from the electrical heating of the sensor to the transient gas temperatures at six distinct temperature stations had to be covered. 
The impact of the non-vanishing thermal mass of the heater and the finite duration of the enthalpy pulse could be implemented by feeding the enthalpy into a virtual cylinder of an adequate virtual thermal diffusivity. This approach sufficiently accurate describes the conversion of electrical power into a short enthalpy pulse that is liberated to the surrounding gas over an interval in time.

The impact of the non-vanishing thermal mass of the resistance thermometers that unexpectedly significantly affects the transport of heat within the active area of the sensor could also be described by virtual cylinders surrounding the line thermometers. The RTDs substantially perturb the otherwise near-cylindrical symmetry of the temperature profile.

The transport of heat from the heater to the temperature stations can effectively be characterized by the composite-wall approach. This conventional approach furnishes varying local overall transport properties of the heat path.

The above theoretical assumptions could experimentally be tested valid by THW and pulse measurements. Regrettably, the respective working equations for an overall thermal diffusivity remain pending for future studies.

The derivation of the semi-ideal model equation was started with the guess of an appropriate solution to the heat conduction equation at hand. The physical quantities of this pulse equation were then replaced by fitting parameters including a variable time exponent. This so-called regression model relation remarkably well approximates the experimental temperature curves. The backcalculation from the regression model to the semi-ideal model to generate relationships between the fitting parameters and the sought thermal quantities of the gas could successfully be managed by the mathematical procedure of reciprocal (termwise) numerical mapping, a prescribed way to assign to each object in one-ordered sequence, e.g., ideal model data, a particular object in another, e.g., fitted data. Here, simple mapping can substitute the incomparably more complicated mathematical tools of Laplace transform and/or convolution.

So far, the semi-ideal model only applies for the sensor when operated with a quiescent gas. The regression model furnishes the required device constants needed to operate the sensor. Future studies have to demonstrate the applicability also for flowing media. Until now, any run on the sensor was evaluated using empirical working equations.

The results obtained here, first and foremost, imply that any redesign of the PTF sensor has to minimize the number of temperature stations. Just the two T050 RTDs are recommended. Here, the remaining four thermometers were intended for study purposes only.

The initial hypothesis that the ideal model of the sensor is incomplete rather than inappropriate could be tested true, analytically, and experimentally.

Funding Open Access funding enabled and organized by Projekt DEAL.

Open Access This article is licensed under a Creative Commons Attribution 4.0 International License, which permits use, sharing, adaptation, distribution and reproduction in any medium or format, as long as you give appropriate credit to the original author(s) and the source, provide a link to the Creative Commons licence, and indicate if changes were made. The images or other third party material in this article 
are included in the article's Creative Commons licence, unless indicated otherwise in a credit line to the material. If material is not included in the article's Creative Commons licence and your intended use is not permitted by statutory regulation or exceeds the permitted use, you will need to obtain permission directly from the copyright holder. To view a copy of this licence, visit http://creativecommons.org/licen ses/by/4.0/.

\section{References}

1. U. Hammerschmidt, C. Sosna, A. Benkert, A. Meier, F. Völklein, Sensors Actuators A Phys. 295, 23 (2019)

2. U. Hammerschmidt, C. Sosna, A. Benkert, A. Meier, F. Völklein, Sensing Imaging (2019). https:// doi.org/10.1007/s11220-019-0264-9

3. H.S. Carslaw, J.C. Jaeger, Conduction of Heat in Solids, 2nd edn. (Clarendon Press, Oxford, 1950)

4. TUV NEL, Physical properties data services (PPDS), Thermodynamic properties database and calculation suite. https:/www.tuvsud.com/en-gb/industries/chemical-and-process/flow-measurement/ consultancy-services/ppds.aa

5. U. Grigull, H. Sandner, Wärmeleitung (Springer, Berlin, 1979)

6. J. Kestin, W. A. Wakeham, Transport Properties of Fluids, Thermal Conductivity, Viscosity, and Diffusion Coefficient, ed. by C.Y. Ho (Hemisphere Publ. Corp., New York, 1990).

7. M.J. Assael, K.D. Antoniadis, W.A. Wakeham, Int. J. Thermophys. 31, 1051 (2010)

8. M.J. Assael, W.A. Wakeham, Thermal conductivity and thermal diffusivity, in Handbook of Experimental Fluid Mechanics, ed. By C. Tropea, A.L. Yaril, J.F. Foss (Springer, Berlin, 2007).

9. K.D. Antoniadis, G.J. Tertsinidou, M.J. Assael, W.A. Wakeham, Int. J. Thermophys. 37, 78 (2016)

10. U. Hammerschmidt, W. Sabuga, Int. J. Thermophys. 21, 1255 (2000)

11. G. Bovesecchi, P. Coppa, S. Corasaniti, M. Potenza, Int. J. Thermophys. 39, 82 (2018)

Publisher's Note Springer Nature remains neutral with regard to jurisdictional claims in published maps and institutional affiliations. 